\title{
Oxymatrine induces cell cycle arrest and apoptosis and suppresses the invasion of human glioblastoma cells through the EGFR/PI3K/Akt/mTOR signaling pathway and STAT3
}

\author{
ZHIBO DAI $^{1,2}$, LIGANG WANG $^{1,2}$, XIAOXIONG WANG $^{1,2}$, BOXIAN ZHAO $^{1,2}$, WENYANG ZHAO $^{1,2}$, \\ SHASHANK SINGH BHARDWAJ ${ }^{1,2}$, JUNYI YE ${ }^{1,2}$, ZHIQIN YIN ${ }^{1,2}$, \\ JUN ZHANG ${ }^{1,2}$ and SHIGUANG ZHAO ${ }^{1,2}$ \\ ${ }^{1}$ Department of Neurosurgery, The First Affiliated Hospital of Harbin Medical University; \\ ${ }^{2}$ Institute of Brain Science, Harbin Medical University, Harbin, Heilongjiang 150001, P.R. China
}

Received October 15, 2017; Accepted June 7, 2018

DOI: $10.3892 /$ or.2018.6512

\begin{abstract}
Oxymatrine (OM), a natural quinolizidine alkaloid extracted from the traditional Chinese herb Sophora flavescens, has been revealed to produce antitumor activities in various cancer cell lines, including glioblastoma lines, in vitro. However, the mechanisms by which OM exerts its antitumor effect against glioma are poorly understood. The aim of this study was to investigate the role of $\mathrm{OM}$ in the proliferation, apoptosis and invasion of glioma cells and to reveal the underlying mechanisms. The effects of OM on U251MG cells in vitro were determined using a Cell Counting Kit-8 (CCK-8) assay, flow cytometric analysis, Annexin V-FITC/PI staining, DAPI staining, a terminal deoxynucleotidyl transferase-mediated dUTP nick end-labeling (TUNEL) assay, a Transwell assay and western blotting. Our data indicated that $\mathrm{OM}$ inhibited proliferation, arrested the cell cycle at the G0/G1 phase, decreased the expression levels of G1 cell cycle regulatory proteins (cyclin D1, CDK4 and CDK6), inhibited invasion and induced apoptosis in glioma cells. Additional investigations revealed that the expression levels of p-STAT3 and key proteins in the EGFR/PI3K/Akt/mTOR signaling pathway, such as p-EGFR, p-Akt and p-mTOR, were markedly decreased after OM treatment, while the total STAT3, EGFR, Akt and mTOR levels were not affected. These findings indicated that the EGFR/PI3K/Akt/mTOR signaling pathway and STAT3 suppression may be a potential mechanism of the
\end{abstract}

Correspondence to: Professor Shiguang Zhao, Department of Neurosurgery, The First Affiliated Hospital of Harbin Medical University, Harbin Medical University, 23 Youzheng Street, Nangang, Harbin, Heilongjiang 150001, P.R. China

E-mail: guangsz@hotmail.com

Key words: oxymatrine, glioma, cell cycle arrest, apoptosis, invasion, EGFR/PI3K/Akt/mTOR signaling, STAT3
OM-mediated antitumor effect in glioblastoma cells and that EGFR may be a target of OM. Hence, OM may be a promising drug and may offer a novel therapeutic strategy for malignant gliomas in the future.

\section{Introduction}

Due to its high metastasis and invasion, glioblastoma is the most common malignant tumor of the central nervous system (CNS) in adults with poor prognoses (1), and a median survival of 10-14 months after the initial diagnosis (2). Accordingly, the recurrence of gliomas remains unavoidable. Currently, early diagnosis and a multimodal approach are the basic requirements for glioma treatments, which include surgical resection, chemotherapeutics and radiotherapy and have an extremely poor prognosis. The major challenges for glioma treatment include many barriers, such as the extent of surgical resection and resistance to radiotherapy and chemotherapy (3). Thus, it is important to explore more efficacious measures against glioma to address the aforementioned issues, and there is an urgent need to develop new compounds against glioma.

Epidermal growth factor receptor (EGFR) is a member of the HER family, which also includes HER2, HER3 and HER4, and EGFR has been identified as an oncogene in many malignant tumors $(4,5)$. EGFR and its downstream signaling pathways are pivotal regulators of many cellular processes, including cell differentiation, metabolism, proliferation and survival, in a large variety of tumors (6-8). EGFR is also overexpressed in most gliomas (9). Therefore, therapies that target EGFR and its downstream signaling pathways may be potential treatments for glioblastoma.

Oxymatrine (OM), a natural quinolizidine alkaloid that is the principal component of the traditional Chinese herb Sophora flavescens, has been reported to produce a great diversity of pharmacological effects, such as anti-inflammatory, antiviral, immunoregulatory and anti-apoptosis effects. Originally, OM was used for the treatment of viral hepatitis (10-14). More recent in vitro studies have revealed 
the significant antitumor activity of OM in the cells of many human cancers, including hepatic $(15)$, pancreatic $(16,17)$, gastric (18) and colorectal carcinoma (19), as well as mastocarcinoma (20). However, the antitumor activity of OM in glioma cells has been rarely studied and the mechanism by which OM exerts its antitumor effect against glioma has not been reported.

In the present study, we studied the effect of OM on glioblastoma cells. We found that OM markedly suppressed the proliferation and invasion, as well as promoted the apoptosis of glioma cells. Further studies revealed that OM arrested the cell cycle at the G0/G1 phase and decreased the protein expression levels of cyclin D1 and CDK4/6. In addition, our findings are the first to demonstrate that the EGFR/PI3K/Akt/mTOR signaling pathway and STAT3 are involved in the OM-induced inhibition in glioma cells. In summary, these results indicated that OM may be a promising antineoplastic agent against gliomas.

\section{Materials and methods}

Reagents. Dulbecco's modified Eagle's medium (DMEM) and fetal bovine serum (FBS) were purchased from Corning Cellgro (Mediatech, Inc., Manassas, VA, USA), and antibiotics (streptomycin and penicillin) were purchased from Corning Incorporated (Corning, NY, USA). OM was purchased from Shanghai Aladdin Bio-Chem Technology Co., Ltd. (Shanghai, China), and the purity (over 98\%) was confirmed by high-performance liquid chromatography (HPLC). OM was dissolved in dimethyl sulfoxide (DMSO; Sigma-Aldrich, St. Louis, MO, USA) at a concentration of $10 \mathrm{mg} / \mathrm{ml}$, stored at $-20^{\circ} \mathrm{C}$, and then freshly diluted with culture medium for each experiment. An Annexin V-FITC Apoptosis Detection kit and a Cycletest Plus DNA Reagent kit were purchased from BD Biosciences (Franklin Lakes, NJ, USA).

Cell culture. The U251MG human malignant glioma cell line was obtained from the Institute of Brain Science of Harbin Medical University (Harbin, China). The cells were cultured in DMEM supplemented with $10 \%$ FBS and $1 \%$ streptomycin/penicillin at $37^{\circ} \mathrm{C}$ in an incubator with a humidified atmosphere of $5 \% \mathrm{CO}_{2}$ and $95 \%$ air.

Cell viability assay. A Cell Counting Kit-8 (CCK-8; Dojindo Molecular Technologies, Inc., Kumamoto, Japan) assay was performed to explore the cytotoxic effects of OM and/or erlotinib on human glioblastoma cells. U251MG cells were seeded in a 96-well plate at a density of 1,000 cells/well and then treated with $\mathrm{OM}$ at various concentrations $(0,0.25,0.5$, 1,2 and $4 \mathrm{mg} / \mathrm{ml}$ ) for 24,48 and $72 \mathrm{~h}$, or treated with $2 \mu \mathrm{M}$ erlotinib in the absence or presence of $1 \mathrm{mg} / \mathrm{ml} \mathrm{OM}$ for $48 \mathrm{~h}$. Following the treatment with OM and/or erlotinib, $10 \mu \mathrm{l}$ of CCK- 8 was added to each well, and the cells were incubated at $37^{\circ} \mathrm{C}$ for $1 \mathrm{~h}$. A microplate reader (Tecan Group Ltd., Männedorf, Switzerland) was used to measure the absorbance of each well at $450 \mathrm{~nm}$ to assess the proliferation.

Detection of cell apoptosis by flow cytometry. An Annexin V-fluorescein isothiocyanate (FITC) assay was employed to quantify apoptotic cells by flow cytometry.
U251MG cells were seeded into 6-well plates at a density of $2 \times 10^{5}$ cells/well, cultured overnight, and then cultured with $\mathrm{OM}$ at a final concentration of $0.5,1$, or $2 \mathrm{mg} / \mathrm{ml}$ or with DMSO for $48 \mathrm{~h}$. Then, the cells were harvested by trypsin, washed with ice-cold PBS twice, collected, fixed and stained using an Annexin V-FITC/PI double fluorescence apoptosis detection kit according to the manufacturer's instructions. The samples were then analyzed by flow cytometry (BD FACSCalibur System; BD Biosciences).

TUNEL assay. DNA fragmentation was performed using a One-Step TUNEL kit (Beyotime Institute of Biotechnology, Shanghai, China) following the manufacturer's recommendations. Briefly, U251MG cells were exposed to OM $(0.5,1$ or $2 \mathrm{mg} / \mathrm{ml}$ ) for $48 \mathrm{~h}$ and then fixed in $4 \%$ paraformaldehyde for $10 \mathrm{~min}$ at room temperature. Subsequently, the cells were washed with PBS three times and permeabilized for 2 min on ice and then the cells were resuspended in TUNEL working solution. Following incubation for $1 \mathrm{~h}$ in a humidified atmosphere at $37^{\circ} \mathrm{C}$ in the dark, the cells were counterstained with DAPI staining solution for $5 \mathrm{~min}$ at room temperature, and then, TUNEL-positive cells were visualized under a fluorescence microscope. DAPI was used for the nuclear staining.

Cell cycle analysis by flow cytometry. The cell cycle phase distribution was evaluated by flow cytometric analysis of the DNA content of cells with the BD Cycletest Plus DNA Reagent kit (BD Biosciences) according to the manufacturer's instructions. In brief, cells were plated in 6-well plates and treated with different concentrations of OM $(0.5,1$ and $2 \mathrm{mg} / \mathrm{ml}$ ) for $48 \mathrm{~h}$, harvested, fixed in $70 \%$ pre-chilled ethanol $\left(-20^{\circ} \mathrm{C}\right)$ and maintained at $4^{\circ} \mathrm{C}$ overnight. Then, the cells were resuspended in propidium iodide (PI) buffer $(50 \mathrm{~g} / \mathrm{ml} \mathrm{PI}$ and $100 \mu \mathrm{g} / \mathrm{ml} \mathrm{RNase}$ ) and incubated for $30 \mathrm{~min}$ shielded from light at room temperature. The cells were then washed twice with PBS. The percentages of cells in each phase of the cell cycle were determined by BD FACSCalibur flow cytometer (BD Biosciences, Fraklin Lakes, NJ, USA) and analyzed by ModFit LT version 4.1 software (Verity Software House, Topsham, ME, USA).

Cell invasion assay. Cell invasion assays were performed using a Corning Matrigel invasion assay system according to the manufacturer's instructions. Following treatment with OM at various concentrations $(0.25,0.5,1$ and $2 \mathrm{mg} / \mathrm{ml})$ for $24 \mathrm{~h}$ or $48 \mathrm{~h}$, cells were seeded for $24 \mathrm{~h}$ and suspended in serum-free DMEM. Then, the cells were placed in the upper part of each chamber, whereas the lower chambers contained FBS (10\%). After incubation in a humidified $5 \% \mathrm{CO}_{2}$ atmosphere at $37^{\circ} \mathrm{C}$ for 24 or $48 \mathrm{~h}$, the cells in the upper chamber and on the Matrigel were mechanically removed with a cotton swab, and the cells on the bottom side of the filter were fixed in $4 \%$ paraformaldehyde for $30 \mathrm{~min}$ at room temperature. Subsequently, the cells were washed with PBS three times and stained with $0.1 \%$ crystal violet for $15 \mathrm{~min}$ at room temperature. Then, the cells were washed three times with PBS and counted under a inverted phase contrast microscope (magnification, x200).

Western blot analysis. Following treatment with different concentrations of $\mathrm{OM}(0.5,1$ and $2 \mathrm{mg} / \mathrm{ml})$, or with $2 \mu \mathrm{M}$ 
erlotinib in the absence or presence of $1 \mathrm{mg} / \mathrm{ml} \mathrm{OM}$ for $48 \mathrm{~h}$, cells were collected, washed with ice-cold PBS and then lysed in lysis buffer [5 mmol/1 EDTA, $40 \mathrm{mmol} / 1$ Tris ( $\mathrm{pH} 8.0$ ), $150 \mathrm{mmol} / 1 \mathrm{NaCl}, 1 \% \mathrm{NP}-40,2 \mathrm{mg} / \mathrm{ml}$ leupeptin, $2 \mathrm{mg} / \mathrm{ml}$ aprotinin, $5 \mathrm{mg} / \mathrm{ml}$ benzamidine and $0.5 \mathrm{mmol} / \mathrm{l}$ phenylmethylsulfonyl fluoride]. The protein concentration was determined using a BCA protein assay kit (Beyotime Biotechnology, Shanghai, China). An equal amount of protein $(60 \mu \mathrm{g})$ from each group was separated using 10\% SDS-polyacrylamide gel electrophoresis (PAGE), transferred to a PVDF membrane (EMD Millipore, Billerica, MA, USA), blocked with 5\% skim milk in TBS-Tween $20(0.05 \%, \mathrm{v} / \mathrm{v})$ for $1 \mathrm{~h}$, and then incubated with primary antibodies at $4^{\circ} \mathrm{C}$ for $16 \mathrm{~h}$. After washing with TBS-T, the membranes were subsequently incubated with horseradish peroxidase-conjugated secondary antibodies for $1 \mathrm{~h}$ at room temperature. GAPDH or $\beta$-actin was used as protein loading controls. After three washes, the target proteins were visualized using an enhanced chemiluminescent (ECL) kit (Thermo Fisher Scientific; Pierce, Rockford, IL, USA). The band intensities were assessed with the Gel-Pro Analyzer Software version 4.0 (Media Cybernetics, Rockville, MD, USA). Primary antibodies against cyclin D1 (1:1,000; cat. no. 2922), CDK4 (1:1,000; cat. no. 12790), CDK6 (1:1,000; cat. no. 13331), p-Akt (Ser473; 1:500; cat. no. 9271), Akt (1:500; cat. no. 9272), STAT3 (1:1,000; cat. no. 12640), p-STAT3 (Ser727; 1:1,000; cat. no. 9134), caspase-3 (1:1,000; cat. no. 9662), Bax (1:1,000; cat. no. 2774), Bcl-2 (1:1,000; cat. no. 2872), GAPDH (1:1,000; cat.no. 2118) and $\beta$-actin (1:1,000; cat. no. 4970) were purchased from Cell Signaling Technology (Danvers, MA, USA). EGFR (1:1,000; cat. no. GTX628887), p-EGFR (Tyr1068; 1:1,000; cat. no. GTX132810), mTOR (1:1,000; cat. no. GTX101557) and p-mTOR (Ser2448; 1:1,000; cat. no. GTX132803) antibodies were purchased from GeneTex Inc. (Irvine, CA, USA). Secondary antibodies goat anti-rabbit IgG HRP (1:5,000; cat. no. ab6721) and goat anti-mouse IgG HRP (1:5,000; cat. no. ab205719) were purchased from Abcam (Cambridge, UK).

Statistical analysis. All statistical analyses were carried out using the SPSS 22.0 statistical software package (IBM Corp., Armonk, NY, USA). Data were expressed as the mean \pm standard deviation (SD). Differences among groups were analyzed by one-way analysis of variance (ANOVA) followed by Student-Newman-Keuls (SNK) multiple comparison test. Differences between two groups were assessed using the Student's t-test. For each measurement, at least three independent experiments were performed. $\mathrm{P}<0.05$ was considered to indicate a statistically significant difference.

\section{Results}

OM potently inhibits the proliferation of glioblastoma cells. The cytotoxic effects of OM on cells were first evaluated by the CCK- 8 cell viability assay. U251MG cells were incubated with $\mathrm{OM}$ at various concentrations for 24,48 and $72 \mathrm{~h}$, and the results revealed that cell viability was reduced in a dose- and time-dependent manner in U251MG cells (Fig. 1).

OM induces cell cycle arrest in glioblastoma cells. To reveal the underlying mechanisms of $\mathrm{OM}$ in the inhibition of the growth of glioblastoma cells, we performed cell cycle analysis. Cells of the glioblastoma cell line U251MG were exposed to different concentrations of OM for $48 \mathrm{~h}$, and flow cytometric analysis was then performed. Our data revealed that OM led to cell accumulation in the G1 phase with a concomitant decrease in the number of cells in the $\mathrm{S}$ phase in a dose-dependent manner (Fig. 2A). The results indicated that the mechanism of the OM-induced suppression of glioblastoma cell viability involved arresting the cell cycle at the G0/G1 phase.

Furthermore, since cyclin D1, CDK4 and CDK6 are the key regulators of the G0/G1 phase of the cell cycle, we performed western blot analysis to determine the expression level of the proteins in OM-treated glioblastoma cells. The results revealed that the protein expression levels of cyclin D1, CDK4 and CDK6 were significantly reduced by OM (Fig. 2B), indicating that $\mathrm{OM}$ arrests glioma cells at the G0/G1 phase by downregulating CDK4, CDK6 and cyclin D1. Collectively, our data revealed that $\mathrm{OM}$ could potently arrest the proliferation of glioblastoma cells.

OM induces the apoptosis of glioblastoma cells. To evaluate whether OM induced the apoptosis of glioma cells, cells were stained with Annexin V-FITC/PI and then analyzed by flow cytometry. The results revealed that after treatment with various concentrations of OM for $48 \mathrm{~h}$, the percentage of apoptotic cells was significantly increased in a dose-dependent manner compared to that of the control cells (Fig. 3A).

Furthermore, as fragmented nuclei are typical characteristics of apoptosis, we performed DAPI staining and a TUNEL assay to detect alterations in the nuclei. The results revealed that after treatment with various concentrations of OM for $48 \mathrm{~h}$, the number of TUNEL-positive cells was significantly higher in the OM-treated cells (Fig. 3B). Furthermore, to reveal the potential mechanism of OM-induced apoptosis in glioma cells, we assessed the protein expression levels of Bax, Bcl-2, and caspase-3, which are important regulators of apoptosis, by western blot analysis. The results revealed that the expression levels of Bax and caspase- 3 were significantly increased, whereas the level of Bcl-2 was decreased after incubation with OM for $48 \mathrm{~h}$, thus leading to an increased ratio of Bax to Bcl-2 (Fig. 3C). Collectively, the results indicated that OM had pro-apoptotic effects on glioblastoma cells.

OM suppresses the invasion of glioblastoma cells. Since invasion ability is one of the pathophysiological features of malignant gliomas, an assay using Matrigel-coated Transwell membranes was performed to evaluate the effects of $\mathrm{OM}$ on invasion. As displayed in Fig. 4, the percentage of invading cells on the lower side of the membrane was decreased in a dose-dependent manner after treatment with OM for $24 \mathrm{~h}$ (Fig. 4B) and $48 \mathrm{~h}$ (Fig. 4A) compared with that in the control group. The results indicated that the invasion of glioblastoma cells could be significantly suppressed by OM.

The EGFR/PI3K/Akt/mTOR signaling pathway is suppressed by $O M$. EGFR amplification is one of the most common genetic alterations in glioblastoma. Gain-of-function of EGFR can lead to upregulation of the PI3K/Akt/mTOR signaling pathway, which is involved in cell survival and proliferation. Therefore, we assessed whether the EGFR/PI3K/Akt/mTOR 


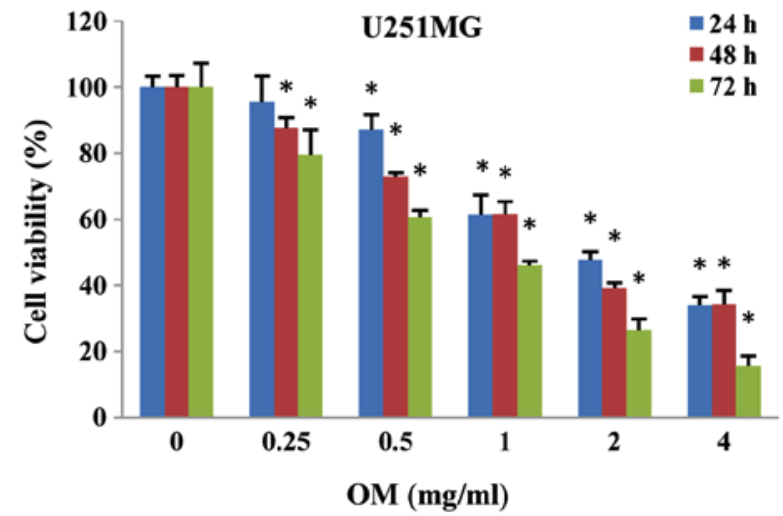

Figure 1. OM inhibits the viability of glioma cells in a dose- and time-dependent manner. U251MG cells were incubated with OM at various concentrations $(0.25-4 \mathrm{mg} / \mathrm{ml})$ or DMSO $(\mathrm{OM} 0 \mathrm{mg} / \mathrm{ml})$ for 24,48 and $72 \mathrm{~h}$. The CCK- 8 assay was used to determine cell viability. The data are presented as the mean $\pm \mathrm{SD}$ of three independent experiments. ${ }^{*} \mathrm{P}<0.05$ compared with the control group (OM $0 \mathrm{mg} / \mathrm{ml})$.

pathway is altered in glioblastoma cells treated with different concentrations of OM for $48 \mathrm{~h}$. As displayed in Fig. 5, OM significantly decreased the expression levels of p-EGFR, p-Akt and p-mTOR in a dose-dependent manner, without affecting total EGFR, Akt and mTOR levels.

Downregulation of the EGFR/PI3K/Akt/mTOR signaling pathway mediates the OM-induced inhibition of proliferation. To further test whether downregulation of the EGFR pathway is responsible for the OM-induced inhibition of glioma cell proliferation, we treated the cells with erlotinib, an inhibitor of EGFR, in the absence or presence of OM for $48 \mathrm{~h}$. The results indicated that in the presence of both erlotinib and OM, the levels of p-EGFR, p-Akt and p-mTOR were significantly lower than those in the presence of erlotinib or OM alone (Fig. 6A). Additionally, compared with the treatment with either erlotinib or OM alone, the combined treatment with erlotinib and OM exhibited a stronger effect that inhibited the proliferation of glioma cells (Fig. 6B).

OM modulates STAT3 in glioblastoma cells. Upregulated STAT3 activity is associated with many human tumors, and STAT3 suppression can mediate tumor regression (21). Therefore, we assessed the expression levels of p-STAT3 and
A

OM (mg/ml) Control

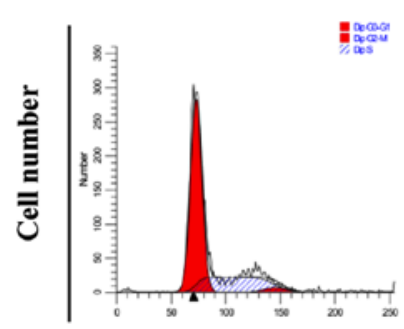

0.5

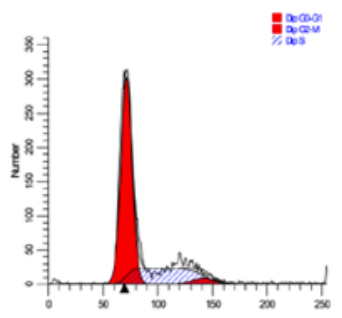

1.0

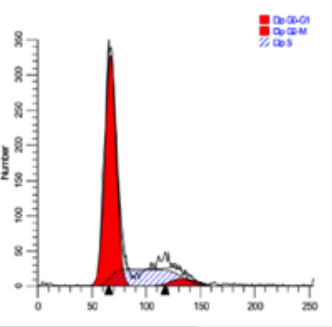

2.0

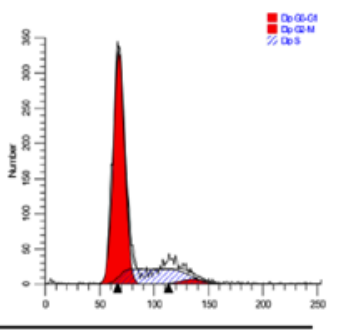

DNA content

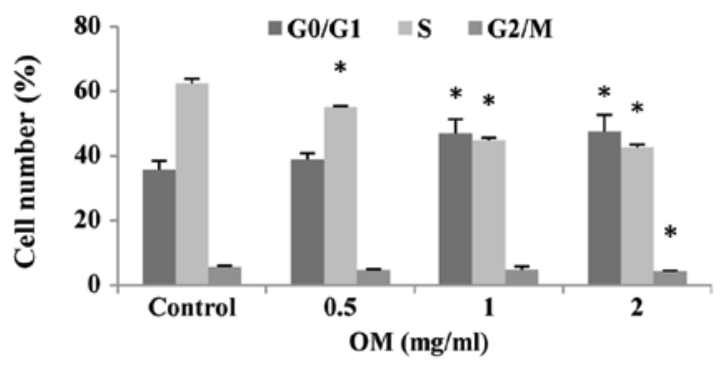

B

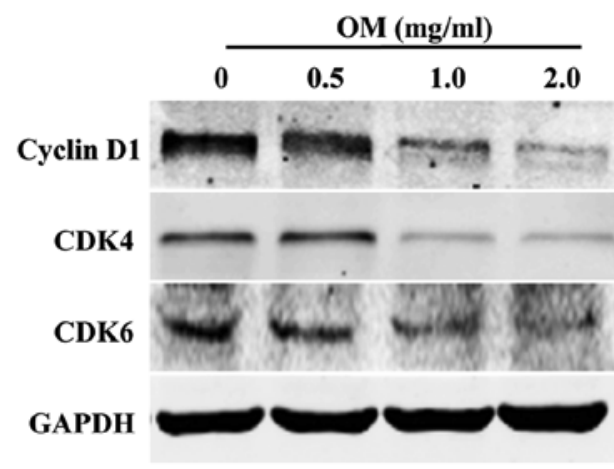

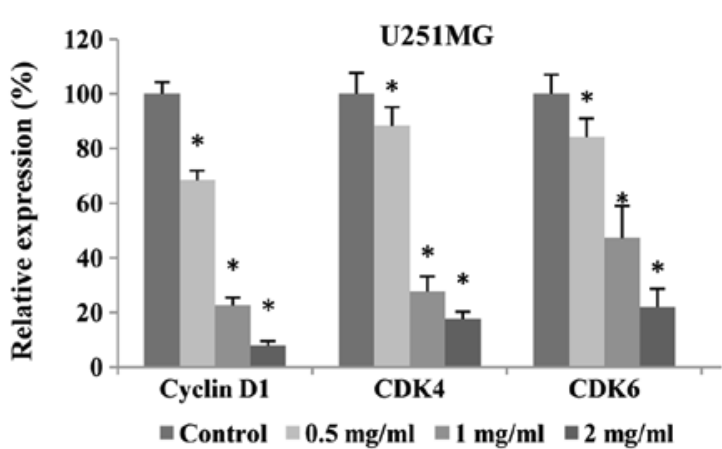

Figure 2. OM induces G1 phase cell-cycle arrest accompanied by suppressed G1 cell-cycle regulatory proteins of glioma cells. U251MG cells were treated with different concentrations of OM $(0.5,1$ and $2 \mathrm{mg} / \mathrm{ml})$ or DMSO (control) for $48 \mathrm{~h}$. (A) Cell cycle distributions analyzed by flow cytometric and statistical analysis of three independent experiments. ${ }^{*} \mathrm{P}<0.05$ compared with the control group. (B) Western blot analysis of G1 cell-cycle regulatory proteins and statistical analysis of three independent experiments. ${ }^{*} \mathrm{P}<0.05$ compared with the control group. 
A
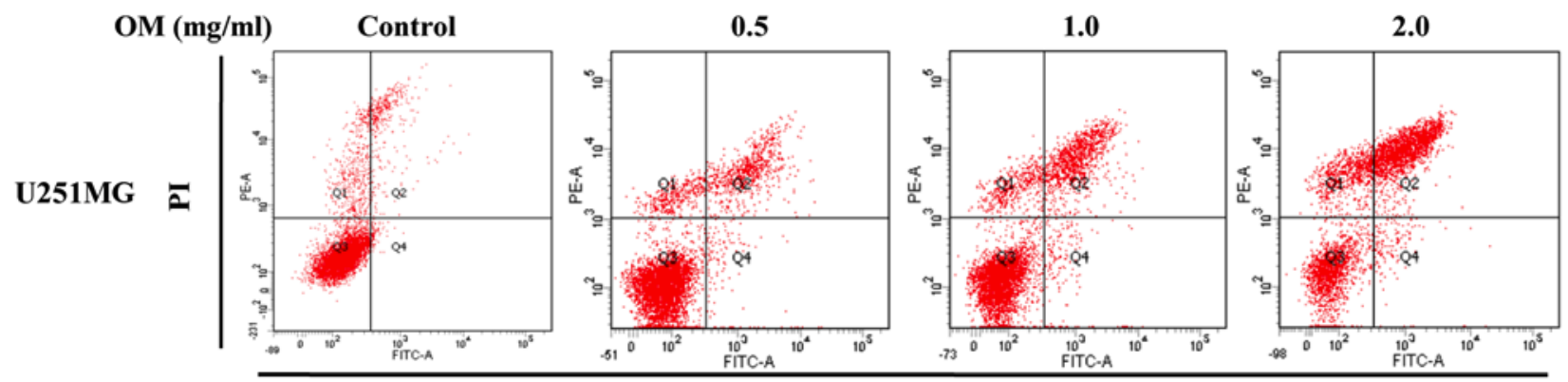

Annexin V-FITC

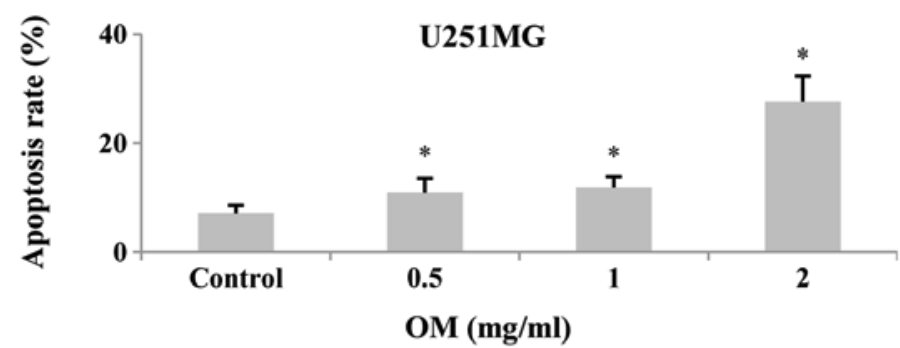

B
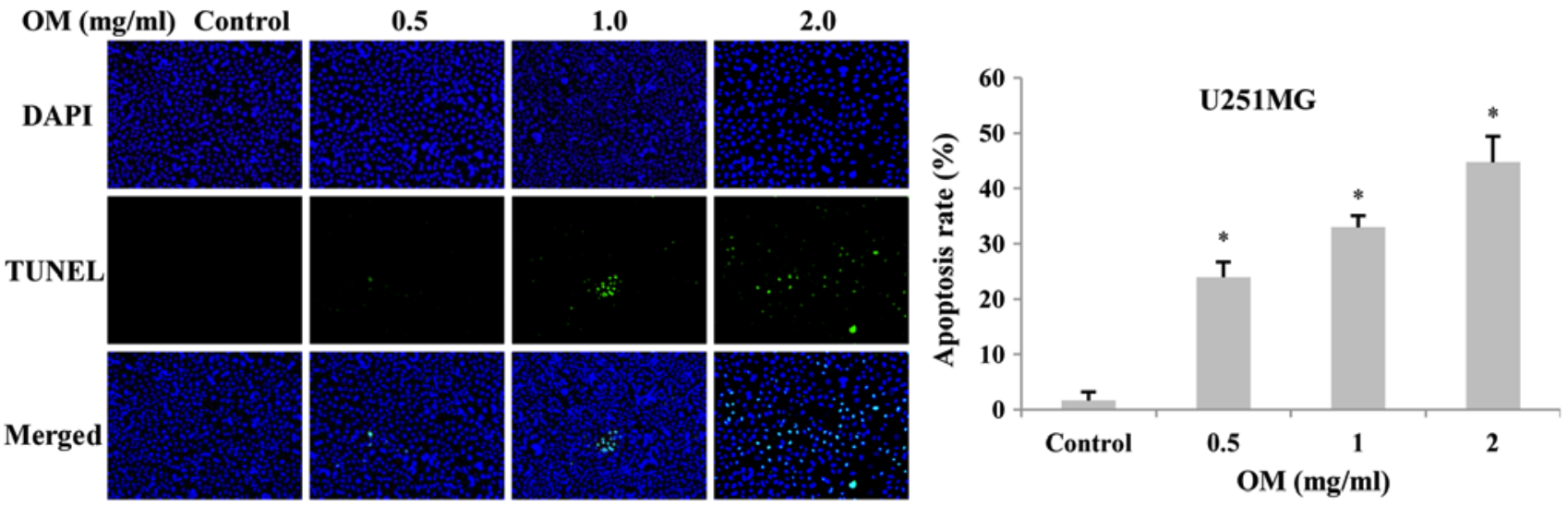

C
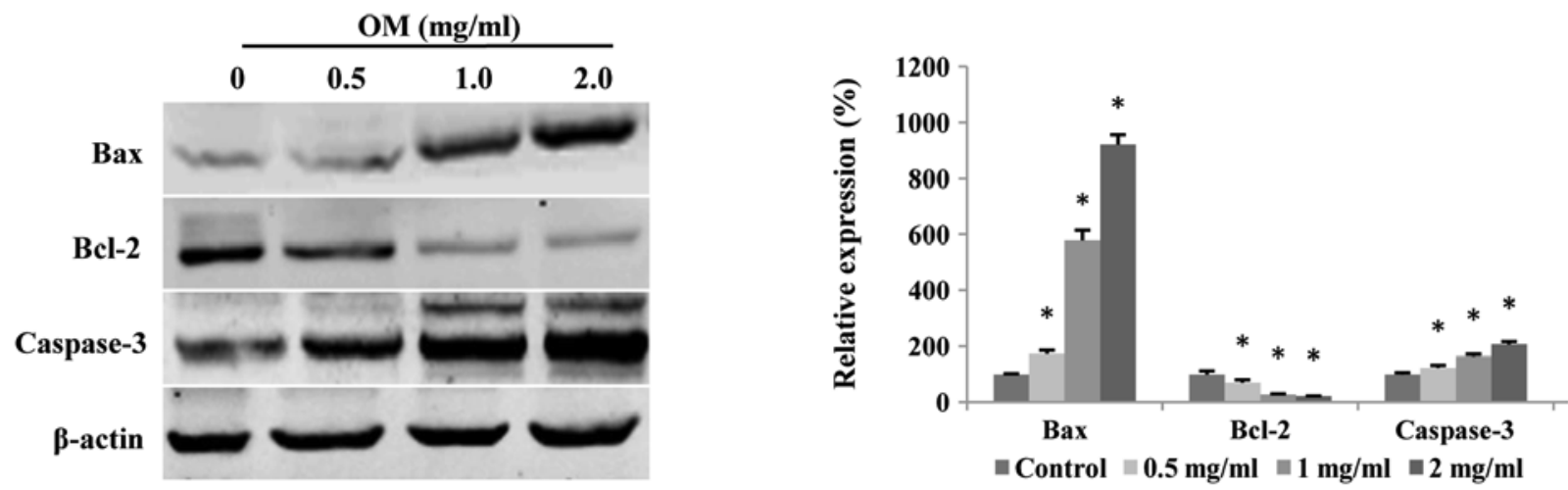

Figure 3. OM induces the apoptosis of glioblastoma cells. U251MG cells were treated with different concentrations of OM $(0.5,1 \mathrm{and} 2 \mathrm{mg} / \mathrm{ml}) \mathrm{or}$ DMSO (control) for $48 \mathrm{~h}$. (A) Analysis of apoptosis via flow cytometry after staining with Annexin V-FITC/PI and statistical analysis of total apoptosis rates including early and late apoptosis $(\mathrm{Q} 2+\mathrm{Q} 4)$ of three independent experiments. "P<0.05 compared with the control group. (B) Apoptotic cells were detected by TUNEL assay. DAPI was used for nuclear staining. Representative images and statistical analysis of three independent experiments are shown. ${ }^{*} \mathrm{P}<0.05$ compared with the control group. (C) The expression levels of apoptosis regulatory proteins were assayed by western blotting. Representatives of three independent experiments are shown. Relative density of apoptosis regulatory proteins is determined by densitometry of the blots. "P<0.05 compared with the control group. 

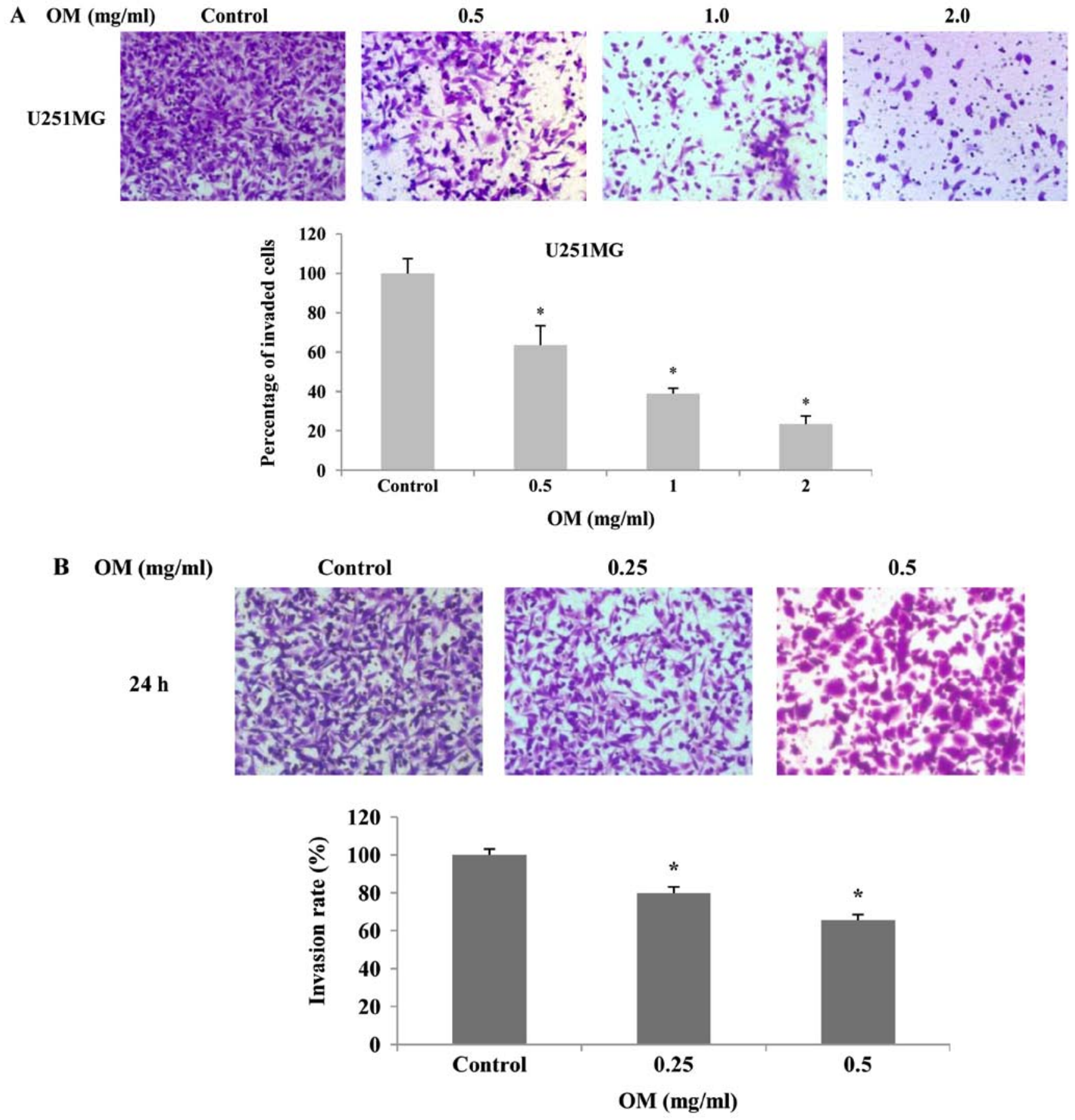

Figure 4. OM suppresses the invasion of glioblastoma cells. (A) Matrigel-coated Transwell assays were used to analyze cell invasion after U251MG cells were treated with different concentrations $(0.5,1 \mathrm{and} 2 \mathrm{mg} / \mathrm{ml})$ of OM or DMSO (control) for $48 \mathrm{~h}$, or (B) with different concentrations $(0.25,0.5 \mathrm{mg} / \mathrm{ml})$ for $24 \mathrm{~h}$. (B) Representatives images of cells that invaded through the Matrigel-coated Transwell inserts are shown. Histogram indicating the percentages of invasive cells compared with the control group. The data are presented as the mean \pm SD of three independent experiments. " $\mathrm{P}<0.05$ compared with the control group.

total STAT3 in glioblastoma cells treated with OM for $48 \mathrm{~h}$. Western blot analysis demonstrated that OM significantly decreased the expression levels of p-STAT3, but did not affect the total STAT3 level (Fig. 7).

\section{Discussion}

Glioblastomas are characterized by aggressive proliferation and high invasion, which play an important role in preventing complete tumor resection, thus leading to tumor recurrence and poor prognosis $(22,23)$. In the present study, our results revealed that $\mathrm{OM}$ exerted a potent antitumor effect against U251MG glioblastoma cells. OM effectively inhibited proliferation, suppressed invasion and induced apoptosis in glioblastoma cells, suggesting that OM may be a potential agent for use in clinics to treat glioma in the future.

The essential attribute of tumorigenesis is uncontrolled and unlimited proliferation, and inhibiting the proliferation of tumor cells can lead to growth arrest. In the present study, we assessed cell viability of glioblastoma cells after 

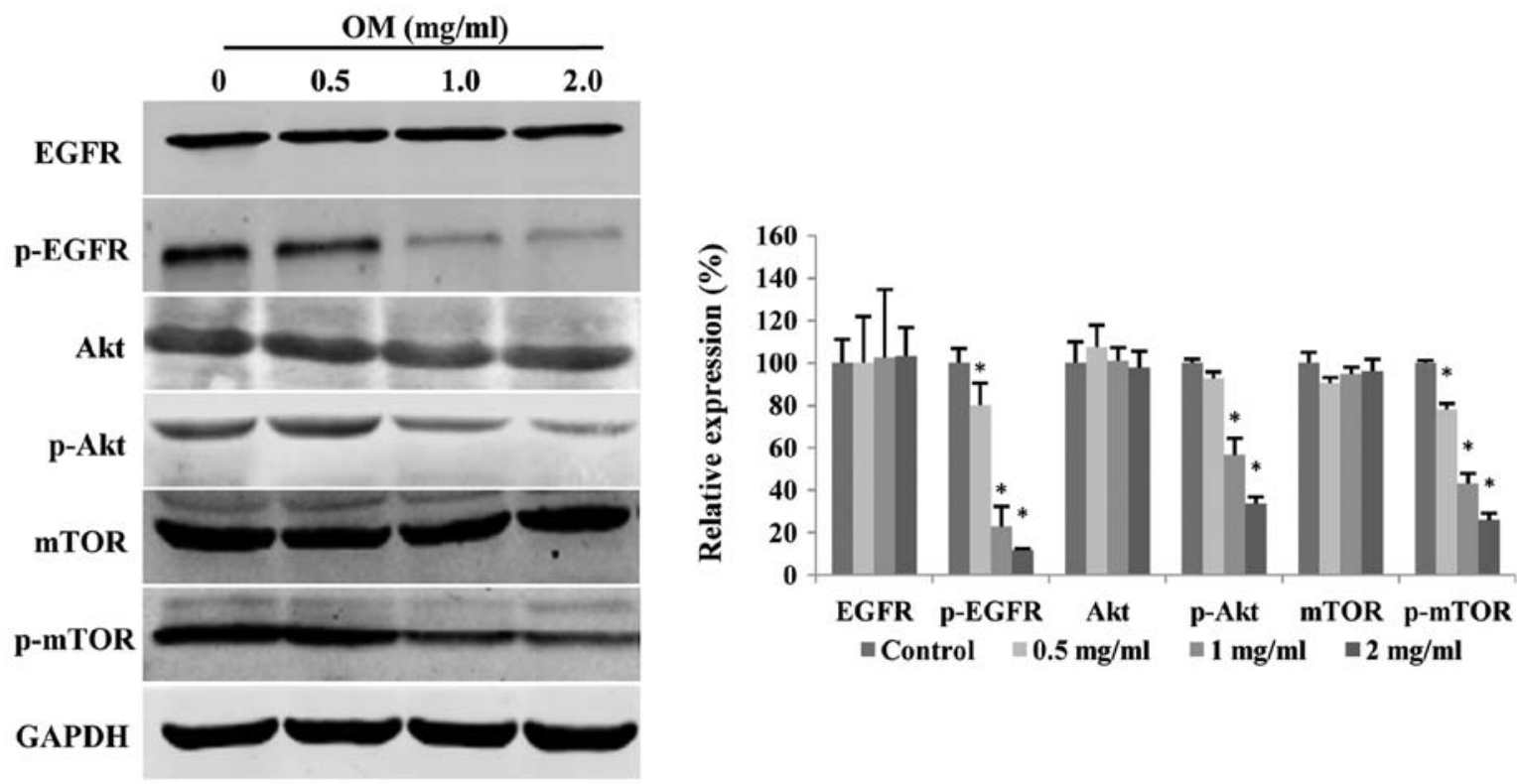

Figure 5. OM inhibits the EGFR/PI3K/Akt/mTOR signalling pathway in glioma cells. U251MG cells were treated with different concentrations of OM (0.5, 1 and $2 \mathrm{mg} / \mathrm{ml}$ ) or DMSO (control) for $48 \mathrm{~h}$. The phosphorylated and total protein levels of EGFR, Akt and mTOR were assayed by western blot analysis. Representatives of three independent experiments are shown. Histogram indicating the quantitative analysis of protein expression from three independent experiments. ${ }^{*} \mathrm{P}<0.05$ compared with the control group.
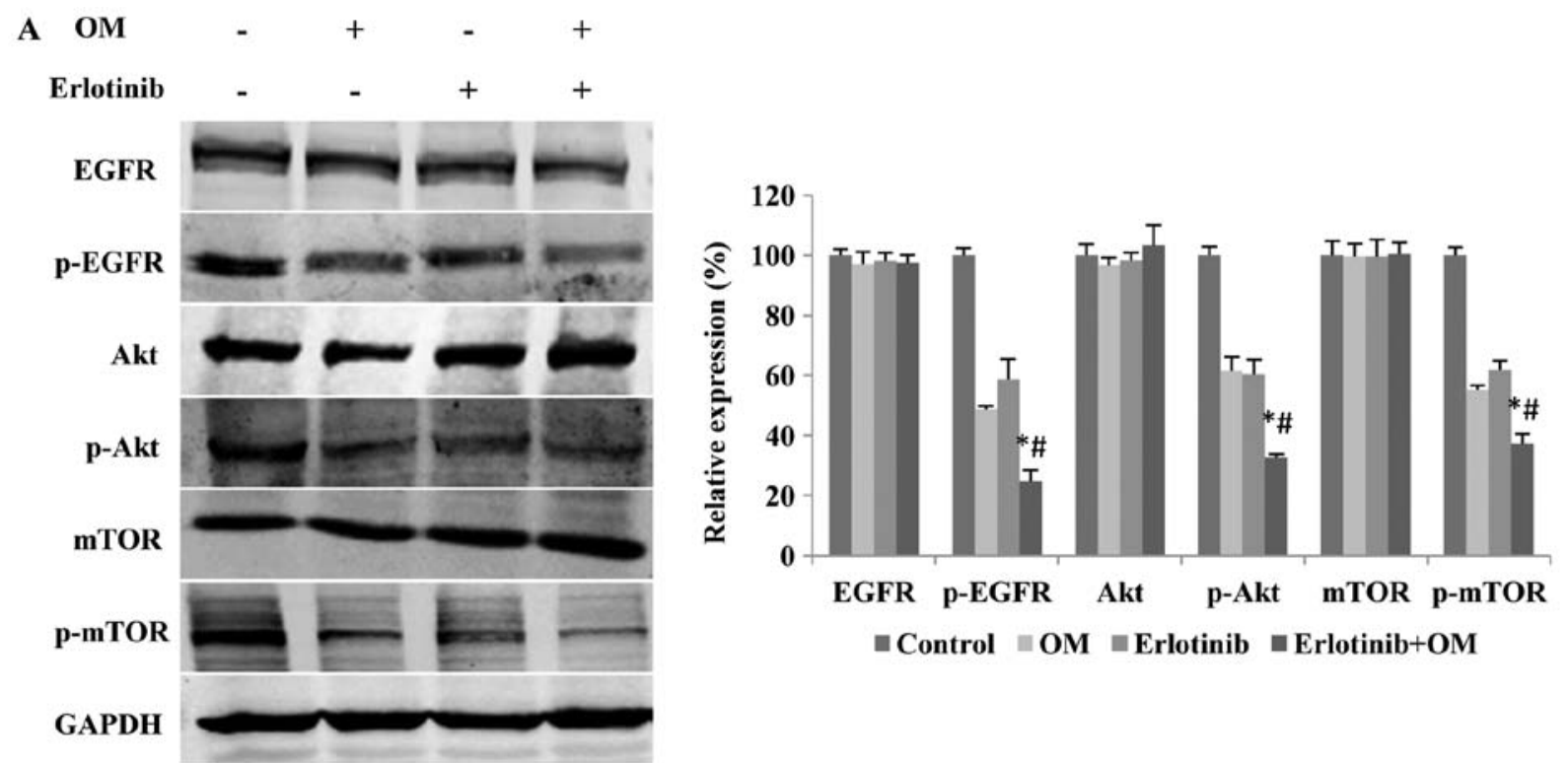

B

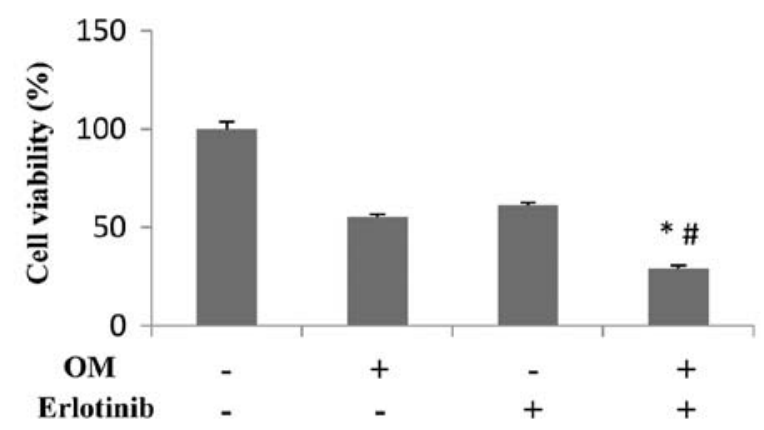

Figure 6. Downregulation of the EGFR/PI3K/Akt/mTOR signaling pathway mediates the OM-induced inhibition of proliferation. U251MG cells were treated with erlotinib $(2 \mu \mathrm{M})$ and OM $(1 \mathrm{mg} / \mathrm{ml})$ either combined or alone for $48 \mathrm{~h}$. (A) Western blot analysis of the key proteins of the EGFR/PI3K/Akt/mTOR signaling pathway and statistical analysis of three independent experiments. ${ }^{*} \mathrm{P}<0.05$ compared with the OM-treated group. ${ }^{\prime \prime} \mathrm{P}<0.05$ compared with the erlotinib-treated group. (B) The cell viabilities assayed by the CCK-8 assay and statistical analysis of three independent experiments. ${ }^{*} \mathrm{P}<0.05$ compared with the OM-treated group. ${ }^{*} \mathrm{P}<0.05$ compared with the erlotinib-treated group. 

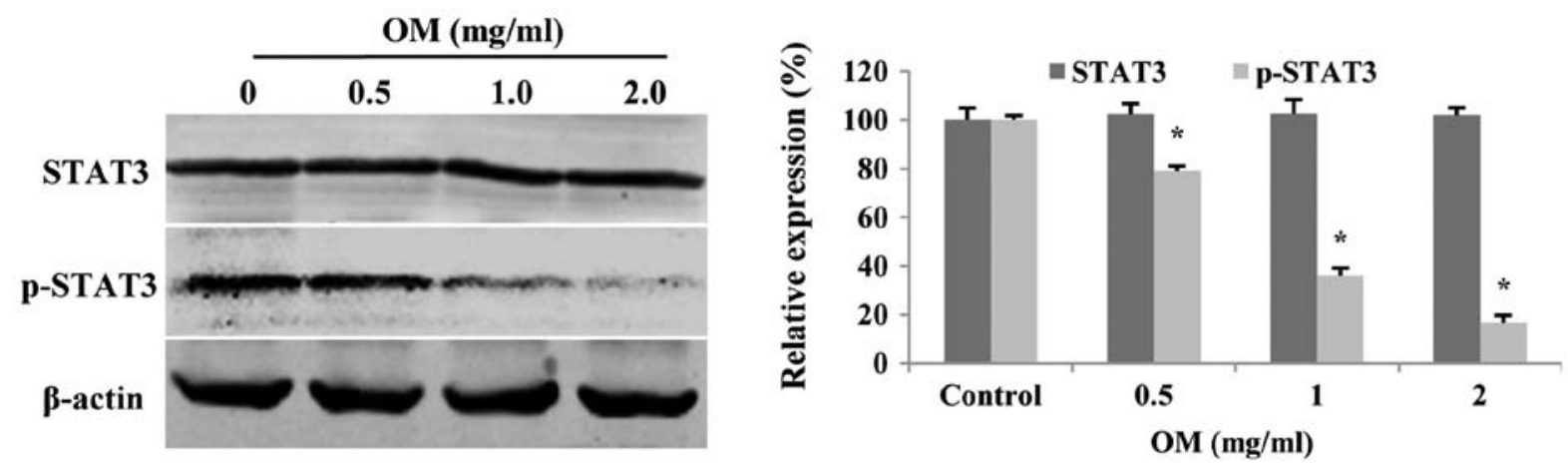

Figure 7. OM modulates STAT3 in glioblastoma cells. After U251MG cells were treated with different concentrations of OM $(0-2 \mathrm{mg} / \mathrm{ml})$ for $48 \mathrm{~h}$, the expression levels of phosphorylated and total STAT3 were assayed by western blot analysis. Representatives of three independent experiments are shown. Histogram indicating the statistical analysis of three independent experiments. ${ }^{*} \mathrm{P}<0.05$ compared with the control group.

incubation with OM. The results revealed that the proliferation of U251MG cells was inhibited in a dose-dependent and time-dependent manner.

EGFR is overexpressed in most human glioblastomas, and it plays a critical role in tumor formation (24). As a result, treatments that particularly aim at EGFR and its downstream pathways in glioblastoma cells may have a potential therapeutic effect. It is well known that the PI3K/Akt/mTOR signaling pathway is regulated by EGFR and is intimately associated with cell growth, autophagy and proliferation. In the present study, key proteins of the EGFR/PI3K/Akt/mTOR signaling pathway, such as p-EGFR, p-Akt and p-mTOR, were inhibited by OM in a dose-dependent manner (Fig. 5). In addition, OM had a similar effect as erlotinib, an EGFR inhibitor, on the EGFR pathway. In combination with erlotinib, OM had a stronger effect that inhibited the proliferation of glioma cells (Fig. 6). These results indicated that $\mathrm{OM}$ is a potential antiproliferative agent against glioblastoma, and the potential mechanism of this antiproliferative effect is interfering with the EGFR/PI3K/Akt/mTOR pathway. In addition, it has been documented that the expression of STAT3 is regulated by mTOR signaling $(25,26)$. In the present study, we determined that the expression level of p-STAT3 was decreased following treatment with OM (Fig. 7), indicating that the effect of OM on glioma cells may be mediated by the EGFR/Akt/mTOR/STAT3 pathway. In addition, dual inhibition of the EGFR/PI3K/Akt/mTOR pathway by erlotinib with OM would also be a reasonable treatment for glioblastoma.

As is well known, dysregulation of cell cycle progression is a basic reason for the abnormal proliferation of tumor cells (27). For cell cycle progression, cell cycle-regulating proteins, such as CDK4, CDK6 and cyclin D1, and their inhibitors play an important role in the $\mathrm{G} 1$ to $\mathrm{S}$ phase transition (28), especially cyclin D1, which regulates this transition. The present study revealed that $\mathrm{OM}$ leads to G0/G1 cell-cycle arrest, which is accompanied by a decrease in the proportion of U251MG cells in the S phase (Fig. 2A). We also observed that $\mathrm{OM}$ decreased the expression level of cyclin D1 and CDK4/6 (Fig. 2B), suggesting that cell cycle arrest may be one of the underlying mechanisms by which OM exerts its antiproliferative effect on glioblastoma cells as a result of the downregulation of positive cell cycle regulatory proteins.
In addition, cyclin D1 is a downstream protein of Akt, which plays an important role in the cell cycle through the G1 phase. The dephosphorylation of EGFR and Akt results in the downregulation of cyclin D1, consequently arresting the cell cycle at the G1 phase (29-32). Consistently, in the present study, we found that OM decreased the expression levels of p-EGFR, p-Akt and cyclin D1 (Figs. 5 and 2B). Similarly, previous studies revealed that OM could induce G1 phase cell-cycle arrest by targeting EGFR in gastric cancer cells (33). Therefore, we hypothesized that the effect of OM on cell cycle arrest in glioblastoma cells may be activated by suppression of the EGFR/Akt/cyclin D1 signaling pathway.

Apoptosis is a type of programmed cell death controlled by genes to maintain the internal environmental stability, and inducing apoptosis should be extremely beneficial for antitumor therapy. Previous studies demonstrated that many antitumor drugs exert therapeutic effects by inducing apoptosis in tumor cells. In the present study, flow cytometry and a TUNEL assay revealed that apoptosis was induced in glioma cells following treatment with OM (Fig. 3A and B), suggesting that $\mathrm{OM}$ inhibits the proliferation of glioblastoma cells by inducing apoptosis.

To investigate potential mechanisms for OM-induced apoptosis, we evaluated the regulators of apoptosis by western blot analysis. As we know, the Bcl-2 family members play a key role in apoptosis such as Bax and Bcl-2, and Bax can promote cell death, while Bcl-2 can inhibit it. Therefore, the $\mathrm{Bax} / \mathrm{Bcl}-2$ ratio determines the susceptibility of tumor cells to drug-mediated apoptosis (34). Furthermore, the caspase family that includes at least 11 members to date, also plays an important role in apoptosis implementing, and caspase-3 due to its characteristic of leading to final disruption of the target cell, is recognized as the key member $(35,36)$. Notably, our study revealed that the Bax/Bcl-2 ratio and caspase-3 increased in glioblastoma cells after treatment with OM (Fig. 3C), which indicate that regulation of the Bcl-2 family protein expression and activation of caspase-3 may lead to OM-induced apoptosis in glioma cells. In addition, the PI3K/Akt/Bcl-2 pathway which can be initiated from EGFR is involved in various cellular processes including cell apoptosis and the activation of $\mathrm{PI} 3 \mathrm{~K} / \mathrm{Akt} / \mathrm{Bcl}-2$ leads to cell survival $(32,37,38)$. In the present study, we found that OM markedly suppressed the expression levels of p-EGFR, p-Akt and Bcl-2 (Figs. 5 and 3C). It is reasonable to 
conclude that $\mathrm{OM}$ induced the apoptosis of glioblastoma cells probably by inactivating the EGFR/PI3K/Akt/Bcl-2 pathway.

Invasion, as one of the main malignant phenotypes of glioblastoma, is a formidable barrier to the treatment of glioblastoma, leading to a poor prognosis. Although distant metastasis of glioma is rare, the pathophysiological features and the ability to invade adjacent areas makes complete surgical resection impossible and increases the risk of tumor recurrence (39-41). Therefore, disrupting the invasion process could be an effective way to treat glioma. Previous studies revealed that $\mathrm{OM}$ interrupted tumor invasion in human gastric cancer, colorectal carcinoma and glioma cells $(33,42,43)$. Consistently, the Transwell results of the present study demonstrated that OM significantly inhibited the invasion of glioblastoma cells in a dose-dependent manner (Fig. 4). However, the exact mechanism by which $\mathrm{OM}$ inhibits invasion warrants further exploration.

In conclusion, OM effectively inhibited the proliferation and invasion of malignant glioma cells and promoted their apoptosis by suppressing the expression of STAT3 and altering the expression of the cell cycle and apoptosis regulators. Furthermore, the EGFR/PI3K/Akt/mTOR pathway is involved in the OM-mediated antitumor effect in glioblastoma cells, and OM combined with erlotinib (a chemotherapeutic agent for tumors) had a more powerful effect that decreased the survival of glioma cells. These findings indicated that the EGFR/PI3K/Akt/mTOR signaling pathway and STAT3 suppression may be a potential mechanism by which OM exerted its antitumor effect against glioma and that EGFR may be the target of OM. Although these results need to be confirmed in vivo, OM, a traditional Chinese herbal product, may be a promising drug and offer a novel therapeutic strategy for malignant gliomas.

\section{Acknowledgements}

The authors thank Dr Zengxiang Dong for providing technical assistance with FACS. We would also like to thank Dr Xiaolei Zhang for helpful discussion. We thank American Journal Experts (AJE) for English language editing.

\section{Funding}

The present study was supported by the Special Fund for Translational Research of Sino-Russia Medical Research Center in Harbin Medical University (nos. CR201410 and CR201512 to S.Z.).

\section{Availability of data and materials}

The datasets used during the present study are available from the corresponding author upon reasonable request.

\section{Authors' contributions}

ZD and SZ designed the research; ZD, LW, XW, BZ, WZ, SSB, JY, ZY and JZ performed the experiments; LW, XW, BZ and $\mathrm{WZ}$ provided reagents and technical support; $\mathrm{ZD}, \mathrm{XW}$ and SZ analyzed the data; ZD and SZ wrote and revised the manuscript. SZ supervised the work. All authors have read and approved the final manuscript. All authors read and approved the manuscript and agree to be accountable for all aspects of the research in ensuring that the accuracy or integrity of any part of the work are appropriately investigated and resolved.

\section{Ethics approval and consent to participate}

Not applicable.

\section{Patient consent for publication}

Not applicable.

\section{Competing interests}

The authors declare that they have no competing interests.

\section{References}

1. Gunther W, Pawlak E, Damasceno R, Arnold H and Terzis AJ: Temozolomide induces apoptosis and senescence in glioma cells cultured as multicellular spheroids. Br J Cancer 88: 463-469, 2003.

2. Louis DN, Ohgaki H, Wiestler OD, Cavenee WK, Burger PC, Jouvet A, Scheithauer BW and Kleihues P: The 2007 WHO classification of tumours of the central nervous system. Acta Neuropathol 114: 97-109, 2007.

3. Saito N, Fu J, Zheng S, Yao J, Wang S, Liu DD, Yuan Y, Sulman EP, Lang FF, Colman H, et al: A high Notch pathway activation predicts response to $\gamma$ secretase inhibitors in proneural subtype of glioma tumor-initiating cells. Stem Cells 32: 301-312, 2014.

4. Hynes NE and Lane HA: ERBB receptors and cancer: The complexity of targeted inhibitors. Nat Rev Cancer 5: 341-354, 2005.

5. Lieto E, Ferraraccio F, Orditura M, Castellano P, Mura AL, Pinto M, Zamboli A, De Vita F and Galizia G: Expression of vascular endothelial growth factor (VEGF) and epidermal growth factor receptor (EGFR) is an independent prognostic indicator of worse outcome in gastric cancer patients. Ann Surg Oncol 15: 69-79, 2008

6. Gusterson BA: Identification and interpretation of epidermal growth factor and c-erbB-2 overexpression. Eur J Cancer 28: 263-267, 1992.

7. Xiao LJ, Lin P, Lin F, Liu X, Qin W, Zou HF, Guo L, Liu W, Wang SJ and Yu XG: ADAM17 targets MMP-2 and MMP-9 via EGFR-MEK-ERK pathway activation to promote prostate cancer cell invasion. Int J Oncol 40: 1714-1724, 2012.

8. Taylor TE, Furnari FB and Cavenee WK: Targeting EGFR for treatment of glioblastoma: Molecular basis to overcome resistance. Curr Cancer Drug Targets 12: 197-209, 2012.

9. Libermann TA, Nusbaum HR, Razon N, Kris R, Lax I, Soreq H, Whittle N, Waterfield MD, Ullrich A and Schlessinger J: Amplification, enhanced expression and possible rearrangement of EGF receptor gene in primary human brain tumours of glial origin. Nature 313: 144-147, 1985.

10. Wang B, Wang G and Xu J: Inhibitory effect of oxymatrine on vascular endothelial cell proliferation induced by tumor. J Pract Oncol 15: 297-300, 2000.

11. Cao YG, Jing S, Li L, Gao JQ, Shen ZY, Liu Y, Xing Y, Wu ML, Wang Y, Xu CQ, et al: Antiarrhythmic effects and ionic mechanisms of oxymatrine from sophora flavescens. Phytother Res 24: 1844-1849, 2010

12. Cui X, Wang Y, Kokudo N, Fang D and Tang W: Traditional Chinese medicine and related active compounds against hepatitis B virus infection. Biosci Trends 4: 39-47, 2010.

13. Deng ZY, Li J, Jin Y, Chen XL and Lu XW: Effect of oxymatrine on the p38 mitogen-activated protein kinases signalling pathway in rats with CCl4 induced hepatic fibrosis. Chin Med J (Engl) 122: 1449-1454, 2009.

14. Fan H, Li L, Zhang X, Liu Y, Yang C, Yang Y and Yin J: Oxymatrine downregulates TLR4, TLR2, MyD88, and NF-kappaB and protects rat brains against focal ischemia. Mediators Inflamm 2009: 704706, 2009. 
15. Ho JW, Ngan Hon PL and Chim WO: Effects of oxymatrine from Ku Shen on cancer cells. Anticancer Agents Med Chem 9: 823-826, 2009.

16. Chen H, Zhang J, Luo J, Lai F, Wang Z, Tong H, Lu D, Bu H, Zhang $\mathrm{R}$ and Lin S: Antiangiogenic effects of oxymatrine on pancreatic cancer by inhibition of the NF- $\mathrm{BB}$-mediated VEGF signaling pathway. Oncol Rep 30: 589-595, 2013.

17. Ling Q, Xu X, Wei X, Wang W, Zhou B, Wang B and Zheng S: Oxymatrine induces human pancreatic cancer PANC-1 cells apoptosis via regulating expression of $\mathrm{Bcl}-2$ and IAP families, and releasing of cytochrome c. J Exp Clin Cancer Res 30: 66, 2011.

18. Song MQ, Zhu JS, Chen JL, Wang L, Da W, Zhu L and Zhang WP: Synergistic effect of oxymatrine and angiogenesis inhibitor NM-3 on modulating apoptosis in human gastric cancer cells. World J Gastroenterol 13: 1788-1793, 2007.

19. Zou J, Ran ZH, Xu Q and Xiao SD: Experimental study of the killing effects of oxymatrine on human colon cancer cell line SW1116. Chin J Dig Dis 6: 15-20, 2005.

20. Zhang Y, Piao B, Zhang Y, Hua B, Hou W, Xu W, Qi X, Zhu X, Pei Y and Lin H: Oxymatrine diminishes the side population and inhibits the expression of $\beta$-catenin in MCF-7 breast cancer cells. Med Oncol 28 (Suppl 1): S99-S107, 2011.

21. Inghirami G, Chiarle R, Simmons WJ, Piva R, Schlessinger K and Levy DE: New and old functions of STAT3: A pivotal target for individualized treatment of cancer. Cell cycle 4: 1131-1133, 2005.

22. Ulrich TA, de Juan Pardo EM and Kumar S: The mechanical rigidity of the extracellular matrix regulates the structure, motility, and proliferation of glioma cells. Cancer Res 69: 4167-4174, 2009.

23. Tu Y, Gao X, Li G, Fu H, Cui D, Liu H, Jin W and Zhang Y: MicroRNA-218 inhibits glioma invasion, migration, proliferation, and cancer stem-like cell self-renewal by targeting the polycomb group gene Bmil. Cancer Res 73: 6046-6055, 2013.

24. Schlegel J, Merdes A, Stumm G, Albert FK, Forsting M, Hynes N and Kiessling M: Amplification of the epidermal-growth-fact or-receptor gene correlates with different growth behaviour in human glioblastoma. Int J Cancer 56: 72-77, 1994.

25. Goncharova EA, Goncharov DA, Damera G, Tliba O, Amrani Y, Panettieri RA Jr and Krymskaya VP: Signal transducer and activator of transcription 3 is required for abnormal proliferation and survival of TSC2-deficient cells: Relevance to pulmonary lymphangioleiomyomatosis. Mol Pharmacol 76: 766-777, 2009.

26. Zhou J, Wulfkuhle J, Zhang H, Gu P, Yang Y, Deng J, Margolick JB, Liotta LA, Petricoin E III and Zhang Y: Activation of the PTEN/mTOR/STAT3 pathway in breast cancer stem-like cells is required for viability and maintenance. Proc Natl Acad Sci USA 104: 16158-16163, 2007.

27. Massagué J: G1 cell-cycle control and cancer. Nature 432 : 298-306, 2004.

28. Kastan MB and Bartek J: Cell-cycle checkpoints and cancer. Nature 432: 316-323, 2004
29. Ye X, Guo Y, Zhang Q, Chen W, Hua X, Liu W, Yang Y and Chen G: $\beta$ Klotho suppresses tumor growth in hepatocellular carcinoma by regulating Akt/GSK-3 $\beta /$ cyclin D1 signaling pathway. PLoS One 8: e55615, 2013.

30. Lu M, Gong X, Lu Y, Guo J, Wang C and Pan Y: Molecular cloning and functional characterization of a cell-permeable superoxide dismutase targeted to lung adenocarcinoma cells. Inhibition cell proliferation through the Akt/p27kip1 pathway. J Biol Chem 281: 13620-13627, 2006.

31. Zhao W, Zhou SF, Zhang ZP, Xu GP, Li XB and Yan JL: Gambogic acid inhibits the growth of osteosarcoma cells in vitro by inducing apoptosis and cell cycle arrest. Oncol Rep 25: 1289-1295, 2011.

32. Pal HC, Sharma S, Strickland LR, Agarwal J, Athar M, Elmets CA and Afaq F: Delphinidin reduces cell proliferation and induces apoptosis of non-small-cell lung cancer cells by targeting EGFR/VEGFR2 signaling pathways. PLoS One 8: e77270, 2013.

33. Guo B, Zhang T, Su J, Wang K and Li X: Oxymatrine targets EGFR $^{\text {p-Tyr845 }}$ and inhibits EGFR-related signaling pathways to suppress the proliferation and invasion of gastric cancer cells. Cancer Chemother Pharmacol 75: 353-363, 2015.

34. Burlacu A: Regulation of apoptosis by Bcl-2 family proteins. J Cell Mol Med 7: 249-257, 2003.

35. Stennicke HR and Salvesen GS: Properties of the caspases. Biochim Biophys Acta 1387: 17-31, 1998.

36. Budihardjo I, Oliver H,Lutter M, Luo X and Wang X: Biochemical pathways of caspase activation during apoptosis. Annu Rev Cell Dev Biol 15: 269-290, 1999.

37. Morozevich GE,Kozlova NI,Ushakova NA,PreobrazhenskayaME and Berman AE: Integrin $\alpha 5 \beta 1$ simultaneously controls EGFR-dependent proliferation and Akt-dependent pro-survival signaling in epidermoid carcinoma cells. Aging 4: 368-374, 2012.

38. Zhang C, Li C, Chen S, Li Z, Jia X, Wang K, Bao J, Liang Y, Wang X, Chen M, et al: Berberine protects against 6-OHDA-induced neurotoxicity in PC12 cells and zebrafish through hormetic mechanisms involving PI3K/AKT/Bcl-2 and Nrf2/HO-1 pathways. Redox Biol 11: 1-11, 2017.

39. Giese A, Bjerkvig R, Berens ME and Westphal M: Cost of migration: Invasion of malignant gliomas and implications for treatment. J Clin Oncol 21: 1624-1636, 2003.

40. Louis DN, Pomeroy SL and Cairncross JG: Focus on central nervous system neoplasia. Cancer Cell 1: 125-128, 2002.

41. Paw I, Carpenter RC, Watabe K, Debinski W and Lo HW Mechanisms regulating glioma invasion. Cancer Lett 362: 1-7, 2015.

42. Wang X, Liu C, Wang J, Fan Y, Wang Z and Wang Y: Oxymatrine inhibits the migration of human colorectal carcinoma RKO cells via inhibition of PAI- 1 and the TGF- $\beta 1 /$ Smad signaling pathway. Oncol Rep 37: 747-753, 2017.

43. Liu F, Wang B, Wang J, Ling X, Li Q, Meng W and Ma J: Oxymatrine inhibits proliferation and migration while inducing apoptosis in human glioblastoma cells. Biomed Res Int 2016: 1784161, 2016. 